\title{
FIXED POINT THEORY FOR VARIOUS CLASSES OF INWARD MULTIVALUED MAPS
}

\author{
RAVI P. AGARWAL ${ }^{\bowtie}$ and DONAL O'REGAN
}

(Received 11 August 2008)

\begin{abstract}
In this paper we present new fixed point theorems for inward and weakly inward type maps between Fréchet spaces. We also discuss Kakutani-Mönch and contractive type maps.
\end{abstract}

2000 Mathematics subject classification: primary 47H10.

Keywords and phrases: fixed point theory, projective limits.

\section{Introduction}

In [11], using multivalued maps $\mathcal{K}_{n}$, we presented new fixed point theorems for Urysohn type maps. In this paper we remove these maps $\mathcal{K}_{n}$ and replace them with a more natural sequentially compact condition. In Section 2 we present new fixed point theorems for weakly inward Kakutani maps and new Leray-Schauder alternatives for inward acyclic and approximable Urysohn type maps and weakly inward Kakutani maps in Fréchet spaces. Also, in Section 2, we discuss KakutaniMönch type operators and contractive maps. The proofs rely on fixed point theory in Banach spaces and viewing a Fréchet space as the projective limit of a sequence of Banach spaces. Our theory is partly motivated by the papers [2-5, 11].

We begin by presenting some definitions and some known facts. Let $X$ and $Y$ be subsets of Hausdorff topological vector spaces $E_{1}$ and $E_{2}$ respectively. We will look at maps $F: X \rightarrow K(Y)$, where $K(Y)$ denotes the family of nonempty compact subsets of $Y$. We say that $F: X \rightarrow K(Y)$ is Kakutani if $F$ is upper semicontinuous with convex values. A nonempty topological space is said to be acyclic if all its reduced Čech homology groups over the rationals are trivial. Now $F: X \rightarrow K(Y)$ is acyclic if $F$ is upper semicontinuous with acyclic values.

Given two open neighbourhoods $U$ and $V$ of the origins in $E_{1}$ and $E_{2}$ respectively, a $(U, V)$-approximate continuous selection of $F: X \rightarrow K(Y)$ is a continuous function $s: X \rightarrow Y$ satisfying

$$
s(x) \in(F[(x+U) \cap X]+V) \cap Y \quad \text { for every } x \in X .
$$

(C) 2009 Australian Mathematical Publishing Association Inc. 0004-9727/2009 \$16.00 
We say that $F: X \rightarrow K(Y)$ is approximable if it is a closed map and if its restriction $\left.F\right|_{K}$ to any compact subset $K$ of $X$ admits a $(U, V)$-approximate continuous selection for every open neighbourhood $U$ and $V$ of the origins in $E_{1}$ and $E_{2}$ respectively.

Let $Q$ be a subset of a Hausdorff topological space $X$ and $x \in X$. The inward set $I_{Q}(x)$ is defined by

$$
I_{Q}(x)=\{x+r(y-x) \mid y \in Q, r \geq 0\} .
$$

If $Q$ is convex and $x \in Q$ then

$$
I_{Q}(x)=x+\{r(y-x) \mid y \in Q, r \geq 1\} .
$$

A mapping $F: Q \rightarrow 2^{X}$ (here $2^{X}$ denotes the family of all nonempty subsets of $X$ ) is said to be weakly inward with respect to $Q$ if $F(x) \cap \overline{I_{Q}(x)} \neq \emptyset$ for all $x \in Q$.

Existence in Section 2 is based on the following continuation theory for $A c A p$ maps. A map is said to be $A c A p$ if it is either acyclic or approximable. In our next definitions $E$ is a Banach space, $C$ a closed convex subset of $E$ and $U_{0}$ a bounded open subset of $E$. We will let $U=U_{0} \cap C$ and $0 \in U$. In our definitions $\bar{U}$ and $\partial U$ denote the closure and the boundary of $U$ in $C$ respectively.

Definition 1.1. We say that $F \in A(\bar{U}, E)$ if $F: \bar{U} \rightarrow K(E)$ is a closed $A c A p$ countably condensing map with $F(x) \subseteq I_{C}(x)$ for all $x \in \bar{U}$.

Definition 1.2. A map $F \in A_{\partial U}(\bar{U}, E)$ if $F \in A(\bar{U}, E)$ with $x \notin F x$ for all $x \in \partial U$.

Definition 1.3. A map $F \in A_{\partial U}(\bar{U}, E)$ is essential in $A_{\partial U}(\bar{U}, E)$ if for every $G \in A_{\partial U}(\bar{U}, E)$ with $\left.G\right|_{\partial U}=\left.F\right|_{\partial U}$ there exists $x \in U$ with $x \in G x$.

The following result was established in [10].

Theorem 1.4. Let $E, C, U_{0}$, and $U$ be as above, $0 \in U$ and $F \in A(\bar{U}, E)$ with

$$
x \notin \lambda F x \quad \text { for all } x \in \partial U \text { and } \lambda \in(0,1] .
$$

Then $F$ is essential in $A_{\partial U}(\bar{U}, E)$.

REMARK 1.5. The proof of Theorem 1.4 is based on the fact that the zero map is essential in $A_{\partial U}(\bar{U}, E)$ and $F \cong 0$ in $A_{\partial U}(\bar{U}, E)$.

If the map $F$ in Theorem 1.4 is Kakutani then in fact we can obtain more general results. The following result can be found in $[6,9]$.

THEOREM 1.6. Let $E$ be a Banach space and $C$ a closed bounded convex subset of $E$. Suppose that $F: C \rightarrow C K(E)$ is an upper semicontinuous condensing map with $F(x) \cap \overline{I_{C}(x)} \neq \emptyset$ for all $x \in C$, where $C K(E)$ denotes the family of nonempty convex compact subsets of $E$. Then $F$ has a fixed point in $E$.

Again in our next definitions $E$ is a Banach space, $C$ a closed convex subset of $E$ and $U_{0}$ a bounded open subset of $E$. We will let $U=U_{0} \cap C$. 
Definition 1.7. We say that $F \in K(\bar{U}, E)$ if $F: \bar{U} \rightarrow C K(E)$ is an upper semicontinuous condensing map with $F(x) \cap \overline{I_{C}(x)} \neq \emptyset$ for all $x \in \bar{U}$.

Definition 1.8. A map $F \in K_{\partial U}(\bar{U}, E)$ if $F \in K(\bar{U}, E)$ with $x \notin F x$ for all $x \in \partial U$.

Definition 1.9. A map $F \in K_{\partial U}(\bar{U}, E)$ is essential in $K_{\partial U}(\bar{U}, E)$ if for every $G \in K_{\partial U}(\bar{U}, E)$ with $\left.G\right|_{\partial U}=\left.F\right|_{\partial U}$ there exists $x \in U$ with $x \in G x$.

Definition 1.10. Two maps $F, G \in K_{\partial U}(\bar{U}, E)$ are homotopic in $K_{\partial U}(\bar{U}, E)$, written $F \cong G$ in $K_{\partial U}(\bar{U}, E)$, if there exists an upper semicontinuous condensing map $N: \bar{U} \times[0,1] \rightarrow C K(E)$ such that $N_{t}(u)=N(t, u): \bar{U} \rightarrow C K(E)$ belongs to $K_{\partial U}(\bar{U}, E)$ for each $t \in[0,1]$ and $N_{0}=F, N_{1}=G$.

The topological transversality theorem for weakly inward Kakutani maps was established in [9].

THEOREM 1.11. Let E, $C, U_{0}$ and $U$ be as above. Suppose that $F$ and $G$ are maps in $K_{\partial U}(\bar{U}, E)$ with $F \cong G$ in $K_{\partial U}(\bar{U}, E)$. Then $F$ is essential in $K_{\partial U}(\bar{U}, E)$ if and only if $G$ is essential in $K_{\partial U}(\bar{U}, E)$.

REMARK 1.12. If the map $F$ in Definition 1.7 (and throughout) is countably condensing instead of condensing then we have to assume that $F(x) \cap I_{C}(x) \neq \emptyset$ for all $x \in \bar{U}$ instead of $F(x) \cap \overline{I_{C}(x)} \neq \emptyset$ for all $x \in \bar{U}$ in Definition 1.7 (and throughout); see [10] for details.

REMARK 1.13. If $0 \in U$ then the zero map is essential in $K_{\partial U}(\bar{U}, E)$; see [10] for details (the proof uses Theorem 1.6).

The following Krasnoselskii type result was established in [9] (there is also an obvious analogue for countably condensing maps if we note Remark 1.12).

THEOREM 1.14. Let $E$ be a Banach space, $C$ a closed convex subset of $E$, and let $W$ and $V$ be open bounded subsets of $E$ with $U_{1}=W \cap C$ and $U_{2}=V \cap C$. Suppose that $0 \in U_{1} \subseteq \bar{U}_{1} \subseteq U_{2}$ and that $F: \bar{U}_{2} \rightarrow C K(E)$ is an upper semicontinuous, condensing map, weakly inward with respect to $C$ (that is, $F(x) \cap \overline{I_{C}(x)} \neq \varnothing$ for all $x \in \bar{U}_{2}$ ). In addition, assume that the following conditions are satisfied:

(i) $x \notin \lambda F x$ for all $x \in \partial U_{2}$ and $\lambda \in[0,1]$;

(ii) there exists $v \in C \backslash\{0\}$ with $x \notin F x+\delta v$ for all $\delta \geq 0$ and $x \in \partial U_{1}$;

(iii) $F(\cdot)+\mu v: \bar{U}_{1} \rightarrow C K(E)$ is a weakly inward map with respect to $C$ for all $\mu \geq 0$ (that is, $[F(x)+\mu v] \cap \overline{I_{C}(x)} \neq \emptyset$ for all $x \in \bar{U}_{1}$ ).

Then $F$ has a fixed point in $U_{2} \backslash U_{1}$.

In this paper we also discuss Mönch type compactness conditions instead of countable condensing. In Section 2 one of our results will be based on a LeraySchauder alternative for Kakutani-Mönch maps [2, 13] which we state here for the convenience of the reader. 
THEOREM 1.15. Let $K$ be a closed convex subset of a Banach space $X$, let $U$ be a relatively open subset of $K, x_{0} \in U$, and suppose that $F: \bar{U} \rightarrow C K(K)$ is an upper semicontinuous map. Also assume that the following conditions hold:

(i) $\quad M \subseteq \bar{U}, M \subseteq \operatorname{co}\left(\left\{x_{0}\right\} \cup F(M)\right)$ with $\bar{M}=\bar{C}$ and $C \subseteq M$ countable, implies that $\bar{M}$ is compact;

(ii) $x \notin(1-\lambda)\left\{x_{0}\right\}+\lambda F x$ for all $x \in \bar{U} \backslash U$ and $\lambda \in(0,1)$.

Then there exists a compact set $\Sigma$ of $\bar{U}$ and an element $x \in \Sigma$ with $x \in F x$.

In Section 2 we will also discuss inward Kakutani-Mönch maps. In our next definition and theorem $E$ is a Banach space, $C$ a closed convex subset of $E$ and $U_{0}$ a bounded open subset of $E$. We will let $U=U_{0} \cap C$ and $0 \in U$. In our definitions $\bar{U}$ and $\partial U$ denote the closure and the boundary of $U$ in $C$ respectively.

Definition 1.16. We say that $F \in K M(\bar{U}, E)$ if $F: \bar{U} \rightarrow C K(E)$ is upper semicontinuous, $F(\bar{U})$ is bounded, $F(x) \subseteq I_{C}(x)$ for all $x \in \bar{U}$; and if $D \subseteq E$ with $D \subseteq \operatorname{co}(\{0\} \cup F(D \cap U))$ and $\bar{D}=\bar{B}$ with $B \subseteq D$ countable then $\overline{D \cap U}$ is compact.

The following theorem $[3,11]$ will be needed in Section 2.

Theorem 1.17. Let $E, C, U_{0}$, and $U$ be as in Definition 1.16, $0 \in U$ and $F \in$ $K M(\bar{U}, E)$, with

$x \notin \lambda F x \quad$ for all $x \in \partial U$ and $\lambda \in(0,1)$.

Then there exists a compact set $\Sigma$ of $\bar{U}$ and an element $x \in \Sigma$ with $x \in F x$.

Finally, in Section 2 we consider contractive type maps. We recall the following two results from the literature [1,8].

THEOREM 1.18 [8, Theorem 3.9]. Let $U$ be an open subset in a Banach space $(X$, $\|\cdot\|)$ and $F: \bar{U} \rightarrow X$. Assume that $0 \in U$ and suppose that there exists a continuous nondecreasing function $\phi:[0, \infty) \rightarrow[0, \infty)$ satisfying $\phi(z)<z$ for all $z>0$ such that $\|F x-F y\| \leq \phi(\|x-y\|)$ for all $x, y \in \bar{U}$. In addition, assume that $F(\bar{U})$ is bounded and $x \neq \lambda F x$ for all $x \in \partial U$ and $\lambda \in(0,1)$. Then $F$ has a fixed point in $\bar{U}$.

TheOREM 1.19 [1, Theorem 2.3 (and Remark 2.1)]. Let $U$ be an open subset in a Banach space $(X,\|\cdot\|)$ and let $F: \bar{U} \rightarrow C(X)$ be a closed map (that is, it has a closed graph), where $C(X)$ denotes the family of nonempty closed subsets of $X$. Assume that $0 \in U$ and suppose that there exists a continuous strictly increasing function $\phi:[0, \infty) \rightarrow[0, \infty)$ satisfying $\phi(z)<z$ for all $z>0$ such that $H(F x, F y) \leq$ $\phi(\|x-y\|)$ for all $x, y \in \bar{U}$. In addition, assume that the following conditions hold:

(i) $\Phi:[0, \infty) \rightarrow[0, \infty)$, given by $\Phi(x)=x-\phi(x)$, is strictly increasing;

(ii) $\Phi^{-1}(a)+\Phi^{-1}(b) \leq \Phi^{-1}(a+b)$ for $a, b \geq 0$;

(iii) $\sum_{i=0}^{\infty} \phi^{i}(t)<\infty$ for $t>0$;

(iv) $\sum_{i=1}^{\infty} \phi^{i}(x-\phi(x)) \leq \phi(x)$ for $x>0$; 
(v) $F(\bar{U})$ is bounded;

(vi) $x \notin \lambda F x$ for all $x \in \partial U$ and $\lambda \in(0,1)$.

Then $F$ has a fixed point in $\bar{U}$.

REMARK 1.20. In fact the assumption that $F$ is closed can be removed in Theorem 1.19. In [1, Theorem 2.3] we assumed a more general contractive condition and the condition is needed there.

Let $(X, d)$ be a metric space and $S$ a nonempty subset of $X$. For $x \in X$ let $d(x, S)=\inf _{y \in S} d(x, y)$. Now suppose that $G: S \rightarrow 2^{X}$. Then $G$ is said to be hemicompact if each sequence $\left\{x_{n}\right\}_{n \in N}$ in $S$ has a convergent subsequence whenever $d\left(x_{n}, G\left(x_{n}\right)\right) \rightarrow 0$ as $n \rightarrow \infty$.

Now let $I$ be a directed set with order $\leq$ and let $\left\{E_{\alpha}\right\}_{\alpha \in I}$ be a family of locally convex spaces. For each $\alpha \in I, \beta \in I$ for which $\alpha \leq \beta$, let $\pi_{\alpha, \beta}: E_{\beta} \rightarrow E_{\alpha}$ be a continuous map. Then the set

$$
\left\{x=\left(x_{\alpha}\right) \in \prod_{\alpha \in I} E_{\alpha}: x_{\alpha}=\pi_{\alpha, \beta}\left(x_{\beta}\right) \forall \alpha, \beta \in I, \alpha \leq \beta\right\}
$$

is a closed subset of $\prod_{\alpha \in I} E_{\alpha}$ which is called the projective limit of $\left\{E_{\alpha}\right\}_{\alpha \in I}$ and is denoted by $\lim _{\leftarrow} E_{\alpha}$ (or $\lim _{\leftarrow}\left\{E_{\alpha}, \pi_{\alpha, \beta}\right\}$ or the generalized intersection [9, p. 439] $\left.\bigcap_{\alpha \in I} E_{\alpha}\right)$.

\section{Fixed point theory in Fréchet spaces}

Let $E=\left(E,\left\{|\cdot|_{n}\right\}_{n \in N}\right)$ be a Fréchet space with the topology generated by a family of seminorms $\left\{|\cdot|_{n} \mid n \in N\right\}$, where $N=\{1,2, \ldots\}$. We assume that the family of seminorms satisfies

$$
|x|_{1} \leq|x|_{2} \leq|x|_{3} \leq \cdots \quad \text { for every } x \in E .
$$

A subset $X$ of $E$ is bounded if for every $n \in N$ there exists $r_{n}>0$ such that $|x|_{n} \leq r_{n}$ for all $x \in X$. For $r>0$ and $x \in E$ we denote $B(x, r)=\{y \in E$ : $|x-y|_{n} \leq r$ for all $\left.n \in N\right\}$. We associate with $E$ a sequence of Banach spaces $\left\{\left(\mathbf{E}_{n},|\cdot|_{n}\right)\right\}$ described as follows. For every $n \in N$ we consider the equivalence relation $\sim_{n}$ defined by

$$
x \sim_{n} y \quad \text { if and only if }|x-y|_{n}=0 .
$$

We denote by $\mathbf{E}^{n}=\left(E / \sim_{n},|\cdot|_{n}\right)$ the quotient space, and by $\left(\mathbf{E}_{n},|\cdot|_{n}\right)$ the completion of $\mathbf{E}^{n}$ with respect to $|\cdot|_{n}$ (the norm on $\mathbf{E}^{n}$ induced by $|\cdot|_{n}$ and its extension to $\mathbf{E}_{n}$ are still denoted by $|\cdot|_{n}$ ). This construction defines a continuous map $\mu_{n}: E \rightarrow \mathbf{E}_{n}$. Now since (2.1) is satisfied the seminorm $|\cdot|_{n}$ induces a seminorm on $\mathbf{E}_{m}$ for every $m \geq n$ (again this seminorm is denoted by $|\cdot|_{n}$ ). Also (2.2) defines an equivalence relation on $\mathbf{E}_{m}$ from which we obtain a continuous map $\mu_{n, m}: \mathbf{E}_{m} \rightarrow \mathbf{E}_{n}$ 
since $\mathbf{E}_{m} / \sim_{n}$ can be regarded as a subset of $\mathbf{E}_{n}$. Now $\mu_{n, m} \mu_{m, k}=\mu_{n, k}$ if $n \leq m \leq k$ and $\mu_{n}=\mu_{n, m} \mu_{m}$ if $n \leq m$. We now assume that the following condition holds:

for each $n \in N$ there exist a Banach space $\left(E_{n},|\cdot|_{n}\right)$

and an isomorphism (between normed spaces) $j_{n}: \mathbf{E}_{n} \rightarrow E_{n}$.

\section{REMARK 2.1.}

(i) For convenience the norm on $E_{n}$ is denoted by $|\cdot|_{n}$.

(ii) In our applications $\mathbf{E}_{n}=\mathbf{E}^{n}$ for each $n \in N$.

(iii) Note that if $x \in \mathbf{E}_{n}$ (or $\mathbf{E}^{n}$ ) then $x \in E$. However, if $x \in E_{n}$ then $x$ is not necessarily in $E$ and in fact $E_{n}$ is easier to use in applications (even though $E_{n}$ is isomorphic to $\mathbf{E}_{n}$ ). For example, if $E=C[0, \infty)$, then $\mathbf{E}^{n}$ consists of the class of functions in $E$ which coincide on the interval $[0, n]$ and $E_{n}=C[0, n]$.

Finally, we assume that

$$
\begin{aligned}
& E_{1} \supseteq E_{2} \supseteq \cdots \quad \text { and } \\
& \quad \text { for each } n \in N,\left|j_{n} \mu_{n, n+1} j_{n+1}^{-1} x\right|_{n} \leq|x|_{n+1} \quad \forall x \in E_{n+1} .
\end{aligned}
$$

(Here we use the notation from [9]; that is, decreasing in the generalized sense.) Let $\lim _{\leftarrow} E_{n}$ (or $\bigcap_{1}^{\infty} E_{n}$ where $\bigcap_{1}^{\infty}$ is the generalized intersection [9]) denote the projective limit of $\left\{E_{n}\right\}_{n \in N}$ (note that $\pi_{n, m}=j_{n} \mu_{n, m} j_{m}^{-1}: E_{m} \rightarrow E_{n}$ for $m \geq n$ ) and note that $\lim _{\leftarrow} E_{n} \cong E$, so for convenience we write $E=\lim _{\leftarrow} E_{n}$.

For each $X \subseteq E$ and each $n \in N$ we set $X_{n}=j_{n} \mu_{n}(X)$, and we let $\bar{X}_{n}$, int $X_{n}$ and $\partial X_{n}$ denote respectively the closure, the interior and the boundary of $X_{n}$ with respect to $|\cdot|_{n}$ in $E_{n}$. Also the pseudo-interior of $X$ is defined by

$$
\text { pseudo-int }(X)=\left\{x \in X \mid j_{n} \mu_{n}(x) \in \bar{X}_{n} \backslash \partial X_{n} \text { for every } n \in N\right\} \text {. }
$$

The set $X$ is pseudo-open if $X=$ pseudo-int $(X)$. For $r>0$ and $x \in E_{n}$ we denote $B_{n}(x, r)=\left\{y \in E_{n}:|x-y|_{n} \leq r\right\}$.

We now show how easily one can extend fixed point theory in Banach spaces to applicable fixed point theory in Fréchet spaces. Our results are motivated by Urysohn type operators. In this case the map $F_{n}$ will be related to $F$ by the closure property (2.10).

TheOREM 2.2. Let $E$ and $E_{n}$ be as described at the beginning of Section 2, $C$ a convex subset in $E, V$ a pseudo-open bounded subset of $E, 0 \in V \cap C, F: Y \rightarrow 2^{E}$ with $Y \subseteq E$, and $\bar{U}_{n}=\overline{V_{n} \cap \bar{C}_{n}} \subseteq Y_{n}$ for each $n \in N$ (here $U_{n}=V_{n} \cap \bar{C}_{n}$ ). Also, for each $n \in N$, assume that $F_{n}: \bar{U}_{n} \rightarrow 2^{E_{n}}$ and suppose that the following conditions are satisfied.

$$
\left\{\begin{array}{l}
\text { For each } n \in\{2,3, \ldots\}, \text { if } y \in U_{n} \text { solves } y \in F_{n} y \text { in } E_{n}, \text { then } \\
j_{k} \mu_{k, n} j_{n}^{-1}(y) \in U_{k} \text { for } k \in\{1, \ldots, n-1\} .
\end{array}\right.
$$


$\left\{\begin{array}{l}\text { For each } n \in N \text {, the map } F_{n}: \bar{U}_{n} \rightarrow K\left(E_{n}\right) \text { is a closed Ac Ap } \\ \text { countably condensing map, where } \bar{U}_{n} \text { denotes the closure of } U_{n} \text { in } \bar{C}_{n} .\end{array}\right.$

For each $n \in N$, we have $F_{n}(x) \subseteq I_{\bar{C}_{n}}(x)$ for all $x \in \bar{U}_{n}$.

$\left\{\begin{array}{l}\text { For each } n \in N, \text { we have } y \notin \lambda F_{n} y \text { in } E_{n} \text { for all } \lambda \in(0,1] \text { and } \\ y \in \partial U_{n} \text {, where } \partial U_{n} \text { denotes the boundary of } U_{n} \text { in } \bar{C}_{n} .\end{array}\right.$

$\left\{\begin{array}{l}\text { For any sequence }\left\{y_{n}\right\}_{n \in N} \text { with } y_{n} \in U_{n} \text { and } y_{n} \in F_{n} y_{n} \text { in } E_{n} \text { for } \\ n \in N \text { and for every } k \in N \text { there exists a subsequence } \\ N_{k} \subseteq\{k+1, k+2, \ldots\}, N_{k} \subseteq N_{k-1} \text { for } k \in\{1,2, \ldots\}, N_{0}=N \text {, and } \\ z_{k} \in \bar{U}_{k} \text { with } j_{k} \mu_{k, n} j_{n}^{-1}\left(y_{n}\right) \rightarrow z_{k} \text { in } E_{k} \text { as } n \rightarrow \infty \text { in } N_{k} .\end{array}\right.$

$\left\{\begin{array}{l}\text { If there exist } w \in Y \text { and a sequence }\left\{y_{n}\right\}_{n \in N} \text { with } y_{n} \in U_{n} \text { and } \\ y_{n} \in F_{n} y_{n} \text { in } E_{n} \text { such that for every } k \in N \text { there exists a subsequence } \\ S \subseteq\{k+1, k+2, \ldots\} \text { of } N \text { with } j_{k} \mu_{k, n} j_{n}^{-1}\left(y_{n}\right) \rightarrow j_{k} \mu_{k}(w) \text { in } E_{k} \\ \text { as } n \rightarrow \infty \text { in } S, \text { then } w \in F w \text { in } E .\end{array}\right.$

Then $F$ has a fixed point in $E$.

REMARK 2.3. Notice that to check (2.9) we need to show that for each $k \in N$ the sequence $\left\{j_{k} \mu_{k, n} j_{n}^{-1}\left(y_{n}\right)\right\}_{n \in N_{k-1}} \subseteq \bar{U}_{k}$ is sequentially compact.

Proof. Fix $n \in N$. We would like to apply Theorem 1.4. To do so we need to show

$$
\bar{C}_{n} \text { is convex }
$$

and

$$
V_{n} \text { is a bounded open subset of } E_{n} \text { and } j_{n} \mu_{n}(0) \in U_{n} \text {. }
$$

First we check (2.11). To see this, let $\hat{x}, \hat{y} \in \mu_{n}(C)$ and $\lambda \in[0,1]$. Then for every $x \in \mu_{n}^{-1}(\hat{x})$ and $y \in \mu_{n}^{-1}(\hat{y})$ we have $\lambda x+(1-\lambda) y \in C$ since $C$ is convex and so $\lambda \hat{x}+(1-\lambda) \hat{y}=\lambda \mu_{n}(x)+(1-\lambda) \mu_{n}(y)$. It is easy to check that $\lambda \mu_{n}(x)+$ $(1-\lambda) \mu_{n}(y)=\mu_{n}(\lambda x+(1-\lambda) y)$ so as a result

$$
\lambda \hat{x}+(1-\lambda) \hat{y}=\mu_{n}(\lambda x+(1-\lambda) y) \in \mu_{n}(C),
$$

and so $\mu_{n}(C)$ is convex. Now since $j_{n}$ is linear, $C_{n}=j_{n}\left(\mu_{n}(C)\right)$ is convex and as a result $\bar{C}_{n}$ is convex. Thus (2.11) holds.

Now since $V$ is pseudo-open and $0 \in V$ then $j_{n} \mu_{n}(0) \in$ pseudo-int $V$ so $j_{n} \mu_{n}(0) \in$ $\bar{V}_{n} \backslash \partial V_{n}$ (here $\bar{V}_{n}$ and $\partial V_{n}$ denote the closure and boundary of $V_{n}$ in $E_{n}$ respectively). Of course

$$
\bar{V}_{n} \backslash \partial V_{n}=\left(V_{n} \cup \partial V_{n}\right) \backslash \partial V_{n}=V_{n} \backslash \partial V_{n}
$$

so $j_{n} \mu_{n}(0) \in V_{n} \backslash \partial V_{n}$, and in particular $j_{n} \mu_{n}(0) \in V_{n}$ (this is easy to see anyway from the definition of $V_{n}$ ). Thus $j_{n} \mu_{n}(0) \in V_{n} \cap \bar{C}_{n}=U_{n}$. Next notice that $V_{n}$ is bounded since $V$ is bounded (note that if $y \in V_{n}$ then there exists $x \in V$ with $y=j_{n} \mu_{n}(x)$ ). It remains to show that $V_{n}$ is open. First notice that $V_{n} \subseteq \bar{V}_{n} \backslash \partial V_{n}$ since if $y \in V_{n}$ then 
there exists $x \in V$ with $y=j_{n} \mu_{n}(x)$, and this together with $V=$ pseudo-int $V$ yields $j_{n} \mu_{n}(x) \in \bar{V}_{n} \backslash \partial V_{n}$; that is, $y \in \bar{V}_{n} \backslash \partial V_{n}$. In addition, notice that

$$
\left.\bar{V}_{n} \backslash \partial V_{n}=\text { int } V_{n} \cup \partial V_{n}\right) \backslash \partial V_{n}=\text { int } V_{n} \backslash \partial V_{n}=\text { int } V_{n}
$$

since int $V_{n} \cap \partial V_{n}=\emptyset$. Consequently,

$$
V_{n} \subseteq \bar{V}_{n} \backslash \partial V_{n}=\text { int } V_{n}, \quad \text { so } V_{n}=\text { int } V_{n} .
$$

As a result $V_{n}$ is open in $E_{n}$. Thus (2.12) holds.

For each $n \in N$ (see Theorem 1.4) there exists $y_{n} \in U_{n}=V_{n} \cap \bar{C}_{n}$ with $y_{n} \in$ $F_{n} y_{n}$. Let us look at $\left\{y_{n}\right\}_{n \in N}$. Notice that $y_{1} \in U_{1}$ and $j_{1} \mu_{1, n} j_{n}^{-1}\left(y_{n}\right) \in U_{1}$ for $k \in\{2,3, \ldots\}$. Now (2.9) with $k=1$ guarantees that there exist a subsequence $N_{1} \subseteq\{2,3, \ldots\}$ and $z_{1} \in \bar{U}_{1}$ with $j_{1} \mu_{1, n} j_{n}^{-1}\left(y_{n}\right) \rightarrow z_{1}$ in $E_{1}$ as $n \rightarrow \infty$ in $N_{1}$. Look at $\left\{y_{n}\right\}_{n \in N_{1}}$. Now $j_{2} \mu_{2, n} j_{n}^{-1}\left(y_{n}\right) \in U_{2}$ for $k \in N_{1}$. Now (2.9) with $k=2$ guarantees that there exist a subsequence $N_{2} \subseteq\{3,4, \ldots\}$ of $N_{1}$ and $z_{2} \in \bar{U}_{2}$ with $j_{2} \mu_{2, n} j_{n}^{-1}\left(y_{n}\right) \rightarrow z_{2}$ in $E_{2}$ as $n \rightarrow \infty$ in $N_{2}$. Note from (2.4) and the uniqueness of limits that $j_{1} \mu_{1,2} j_{2}^{-1} z_{2}=z_{1}$ in $E_{1}$ since $N_{2} \subseteq N_{1}$ (note that $j_{1} \mu_{1, n} j_{n}^{-1}\left(y_{n}\right)=$ $j_{1} \mu_{1,2} j_{2}^{-1} j_{2} \mu_{2, n} j_{n}^{-1}\left(y_{n}\right)$ for $\left.n \in N_{2}\right)$. Proceed inductively to obtain subsequences of integers

$$
N_{1} \supseteq N_{2} \supseteq \cdots \quad \text { where } N_{k} \subseteq\{k+1, k+2, \ldots\}
$$

and $z_{k} \in \bar{U}_{k}$ with $j_{k} \mu_{k, n} j_{n}^{-1}\left(y_{n}\right) \rightarrow z_{k}$ in $E_{k}$ as $n \rightarrow \infty$ in $N_{k}$. Note that $j_{k} \mu_{k, k+1} j_{k+1}^{-1} z_{k+1}=z_{k}$ in $E_{k}$ for $k \in\{1,2, \ldots\}$.

Fix $k \in N$. Note that

$$
\begin{aligned}
z_{k} & =j_{k} \mu_{k, k+1} j_{k+1}^{-1} z_{k+1}=j_{k} \mu_{k, k+1} j_{k+1}^{-1} j_{k+1} \mu_{k+1, k+2} j_{k+2}^{-1} z_{k+2} \\
& =j_{k} \mu_{k, k+2} j_{k+2}^{-1} z_{k+2}=\cdots=j_{k} \mu_{k, m} j_{m}^{-1} z_{m}=\pi_{k, m} z_{m}
\end{aligned}
$$

for every $m \geq k$. We can do this for each $k \in N$. As a result $y=\left(z_{k}\right) \in \lim _{\leftarrow} E_{n}=E$ and also note that $y \in Y$ since $z_{k} \in \bar{U}_{k} \subseteq Y_{k}$ for each $k \in N$. Also since $y_{n} \in F_{n} y_{n}$ in $E_{n}$ for $n \in N_{k}$ and $j_{k} \mu_{k, n} j_{n}^{-1}\left(y_{n}\right) \rightarrow z_{k}=y$ in $E_{k}$ as $n \rightarrow \infty$ in $N_{k}$, we have from (2.10) that $y \in F y$ in $E$.

REMARK 2.4. From the proof we see that condition (2.5) can be removed from the statement of Theorem 2.2. We include it only to explain condition (2.9) (see Remark 2.3).

REMARK 2.5. Note that we could replace $\bar{U}_{n} \subseteq Y_{n}$ above with $\bar{U}_{n}$ a subset of the closure of $Y_{n}$ in $E_{n}$ if $Y$ is a closed subset of $E$ (so in this case we can take $Y=C \cap \bar{V}$ if $\overline{\bar{C}}_{n} \cap V_{n}$ is a subset of the closure of $j_{n} \mu_{n}(C \cap \bar{V})$ in $E_{n}$ and if $C$ is closed). To see this, note that $z_{k} \in \bar{U}_{k}, y=\left(z_{k}\right) \in \lim _{\leftarrow} E_{n}=E$ and $\pi_{k, m}\left(y_{m}\right) \rightarrow z_{k}$ in $E_{k}$ as $m \rightarrow \infty$ and we can conclude that $y \in \bar{Y}=Y$ (note that $q \in \bar{Y}$ if and only if for every $k \in N$ there exists $\left(x_{k, m}\right) \in Y, x_{k, m}=\pi_{k, n}\left(x_{n, m}\right)$ for $n \geq k$ with $x_{k, m} \rightarrow j_{k} \mu_{k}(q)$ in $E_{k}$ as $m \rightarrow \infty)$. 
REMARK 2.6. Suppose that in Theorem 2.2 we replace (2.9) with the following.

$$
\left\{\begin{array}{l}
\text { For any sequence }\left\{y_{n}\right\}_{n \in N} \text { with } y_{n} \in U_{n} \text { and } y_{n} \in F_{n} y_{n} \text { in } E_{n} \text { for } \\
n \in N \text { and for every } k \in N \text { there exist a subsequence } \\
N_{k} \subseteq\{k+1, k+2, \ldots\}, \text { where } N_{k} \subseteq N_{k-1} \text { for } k \in\{1,2, \ldots\}, \text { where } \\
N_{0}=N \text {, and } z_{k} \in U_{k} \text { with } j_{k} \mu_{k, n} j_{n}^{-1}\left(y_{n}\right) \rightarrow z_{k} \text { in } E_{k} \text { as } n \rightarrow \infty \\
\text { in } N_{k} \text {. }
\end{array}\right.
$$

In addition, we assume that $F: Y \rightarrow 2^{E}$ with $\bar{U}_{n} \subseteq Y_{n}$ for each $n \in N$ is replaced by $F: Y \rightarrow 2^{E}$ with $U_{n} \subseteq Y_{n}$ for each $n \in N$. Then the result in Theorem 2.2 is again true.

The proof follows the reasoning in Theorem 2.2 except in this case $z_{k} \in U_{k}$.

Next we present a result for weakly inward Kakutani maps using Theorem 1.6.

THEOREM 2.7. Let $E$ and $E_{n}$ be as described at the beginning of Section 2, $C$ a convex bounded subset in $E, F: Y \rightarrow 2^{E}$ with $Y \subseteq E$, and $\bar{C}_{n} \subseteq Y_{n}$ for each $n \in N$. Also for each $n \in N$ assume that $F_{n}: \bar{C}_{n} \rightarrow 2^{E_{n}}$ and suppose that the following conditions are satisfied.

$\left\{\begin{array}{l}\text { For each } n \in\{2,3, \ldots\}, \text { if } y \in \bar{C}_{n} \text { solves } y \in F_{n} y \text { in } E_{n} \text {, then } \\ j_{k} \mu_{k, n} j_{n}^{-1}(y) \in \bar{C}_{k} \text { for } k \in\{1, \ldots, n-1\} .\end{array}\right.$

$\left\{\begin{array}{l}\text { For each } n \in N \text {, the map } F_{n}: \bar{C}_{n} \rightarrow C K\left(E_{n}\right) \text { is upper semicontinuous } \\ \text { and condensing. }\end{array}\right.$

For each $n \in N, F_{n}(x) \cap \overline{\bar{I}_{n}(x)} \neq \emptyset$ for $x \in \bar{C}_{n}$.

For any sequence $\left\{y_{n}\right\}_{n \in N}$ with $y_{n} \in \bar{C}_{n}$ and $y_{n} \in F_{n} y_{n}$ in $E_{n}$ for

$n \in N$ and for every $k \in N$ there exist a subsequence

$N_{k} \subseteq\{k+1, k+2, \ldots\}$, where $N_{k} \subseteq N_{k-1}$ for $k \in\{1,2, \ldots\}$,

$N_{0}=N$, and $z_{k} \in \bar{C}_{k}$ with $j_{k} \mu_{k, n} j_{n}^{-1}\left(y_{n}\right) \rightarrow z_{k}$ in $E_{k}$ as $n \rightarrow \infty$ in $N_{k}$.

$\left\{\begin{array}{l}\text { If there exist } w \in Y \text { and a sequence }\left\{y_{n}\right\}_{n \in N} \text { with } y_{n} \in \bar{C}_{n} \text { and } \\ y_{n} \in F_{n} y_{n} \text { in } E_{n} \text { such that for every } k \in N \text { there exists a subsequence } \\ S \subseteq\{k+1, k+2, \ldots\} \text { of } N \text { with } j_{k} \mu_{k, n} j_{n}^{-1}\left(y_{n}\right) \rightarrow j_{k} \mu_{k}(w) \text { in } E_{k} \\ \text { as } n \rightarrow \infty \text { in } S, \text { then } w \in F w \text { in } E .\end{array}\right.$

Then $F$ has a fixed point in $E$.

PROOF. For each $n \in N$ there exists (Theorem 1.6) $y_{n} \in \bar{C}_{n}$ with $y_{n} \in F_{n} y_{n}$ in $E_{n}$. Essentially the same reasoning as in Theorem 2.2 establishes the result.

REMARK 2.8. Condition (2.13) can be removed from the statement of Theorem 2.7.

REMARK 2.9. Note that we could replace $\bar{C}_{n} \subseteq Y_{n}$ above with $\bar{C}_{n}$ a subset of the closure of $Y_{n}$ in $E_{n}$ if $Y$ is a closed subset of $E$ (so in this case we can take $Y=C$ if $C$ is a closed subset of $E$ ). 
For our next definitions $E$ and $E_{n}$ are as described at the beginning of Section 2, $C$ is a convex subset of $E, V$ a bounded pseudo-open subset of $E$ and $F: Y \rightarrow 2^{E}$

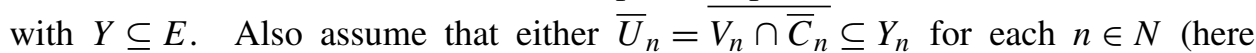
$U_{n}=V_{n} \cap \bar{C}_{n}$ ) or $\bar{U}_{n}$ is a subset of the closure of $Y_{n}$ in $E_{n}$ for each $n \in N$ (with $Y$ a closed subset of $E$ ). In addition, assume for each $n \in N$ that $F_{n}: \bar{U}_{n} \rightarrow 2^{E_{n}}$.

Definition 2.10. $F \in K(Y, E)$ if, for each $n \in N, F_{n} \in K\left(\bar{U}_{n}, E_{n}\right)$ (that is, for each $n \in N, F_{n}: \bar{U}_{n} \rightarrow C K\left(E_{n}\right)$ is an upper semicontinuous condensing map with $F_{n}(x) \cap \overline{I_{\bar{C}_{n}}(x)} \neq \emptyset$ for all $\left.x \in \bar{U}_{n}\right)$, where $\bar{U}_{n}$ denotes the closure of $U_{n}$ in $\bar{C}_{n}$.

Definition 2.11. $F \in K_{\partial}(Y, E)$ if $F \in K(Y, E)$ and, for each $n \in N, x \notin F_{n}(x)$ for all $x \in \partial U_{n}$, where $\partial U_{n}$ denotes the boundary of $U_{n}$ in $\bar{C}_{n}$.

Definition 2.12. A map $F \in K_{\partial}(Y, E)$ is essential in $K_{\partial}(Y, E)$ if, for each $n \in N$, $F_{n} \in K_{\partial U_{n}}\left(\bar{U}_{n}, E_{n}\right)$ is essential in $K_{\partial U_{n}}\left(\bar{U}_{n}, E_{n}\right)$ (that is, for each $n \in N$, every map $G \in K_{\partial U_{n}}\left(\bar{U}_{n}, E_{n}\right)$ with $\left.G\right|_{\partial U_{n}}=\left.F_{n}\right|_{\partial U_{n}}$ has a fixed point in $\left.\bar{U}_{n} \backslash \partial U_{n}\right)$.

REMARK 2.13. Note that if $j_{n} \mu_{n}(0) \in U_{n}$ for each $n \in N$ then $0 \in K_{\partial}(Y, E)$ is essential in $K_{\partial}(Y, E)$ by Remark 1.13.

Definition 2.14. (We assume that $j_{n} \mu_{n}(0) \in U_{n}$ for each $n \in N$.) The maps $F$, $0 \in K_{\partial}(Y, E)$ are homotopic in $K_{\partial}(Y, E)$, written $F \cong 0$ in $K_{\partial}(Y, E)$, if, for each $n \in N, F_{n} \cong j_{n} \mu_{n}(0)$ in $K_{\partial U_{n}}\left(\bar{U}_{n}, E_{n}\right)$.

Theorem 2.15. Let $E$ and $E_{n}$ be as described at the beginning of Section 2, $C$ a convex subset in $E, V$ a bounded pseudo-open subset of $E$ and $F: Y \rightarrow 2^{E}$ with $Y \subseteq$ E. Also assume that either $\bar{U}_{n}=\overline{V_{n} \cap \bar{C}_{n}} \subseteq Y_{n}$ for each $n \in N$ (here $U_{n}=V_{n} \cap \bar{C}_{n}$ ) or $\bar{U}_{n}$ is a subset of the closure of $Y_{n}$ in $E_{n}$ for each $n \in N$ (with $Y$ a closed subset of $E$ ). Suppose that $0 \in V \cap C$ and for each $n \in N$ assume that $F_{n}: \bar{U}_{n} \rightarrow 2^{E_{n}}$ and also suppose that $F \in K_{\partial}(Y, E)$ with (2.5) and that the following condition satisfied.

$$
F \cong 0 \quad \text { in } K_{\partial}(Y, E)
$$

Also assume that (2.9) and (2.10) hold. Then F has a fixed point in E.

Proof. Fix $n \in N$. Remark 2.13 guarantees that the zero map (that is, $G(x)=$ $\left.j_{n} \mu_{n}(0)\right)$ is essential in $K_{\partial U_{n}}\left(\bar{U}_{n}, E_{n}\right)$ for each $n \in N$. Now Theorem 1.11 guarantees that $F_{n}$ is essential in $K_{\partial U_{n}}\left(\bar{U}_{n}, E_{n}\right)$ so in particular there exists $y_{n} \in U_{n}$ with $y_{n} \in$ $F_{n} y_{n}$. Essentially the same reasoning as in Theorem 2.2 (with Remark 2.5) establishes the result.

REMARK 2.16. Condition (2.5) can be removed from the statement of Theorem 2.15.

REMARK 2.17. If for each $n \in N$ the map $F_{n}: \bar{U}_{n} \rightarrow C K\left(E_{n}\right)$ is countably condensing instead of condensing in Definition 2.10 (and throughout) then we assume that $F_{n}(x) \cap I_{\bar{C}_{n}}(x) \neq \emptyset$ for all $x \in \bar{U}_{n}$ instead of $F_{n}(x) \cap \overline{I_{\bar{C}_{n}}(x)} \neq \emptyset$ for all $x \in \bar{U}_{n}$ in Definition 2.10 (and throughout). 
REMARK 2.18. Notice that $0 \in V \cap C$ and (2.18) could be replaced by $F \cong G$ in $K_{\partial}(Y, E)$ (of course we assume that $G \in K_{\partial}(Y, E)$ and we must specify $G_{n}$ for $n \in N$ here).

REMARK 2.19. Remark 2.6 also holds in this situation.

THEOREM 2.20. Let $E$ and $E_{n}$ be as described at the beginning of Section 2, $C$ a convex subset in $E, V$ a bounded pseudo-open subset of $E$ and $F: Y \rightarrow 2^{E}$ with

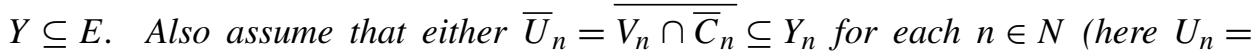
$V_{n} \cap \bar{C}_{n}$ ) or $\bar{U}_{n}$ is a subset of the closure of $Y_{n}$ in $E_{n}$ for each $n \in N$ (with $Y$ a closed subset of $E$ ). Suppose that $0 \in V \cap C$ and for each $n \in N$ assume that $F_{n}: \bar{U}_{n} \rightarrow 2^{E_{n}}$ and also suppose that $F \in K_{\partial}(Y, E)$ with (2.5), (2.9), (2.10) and that the following condition satisfied:

for each $n \in N$, we have $y \notin \lambda F_{n} y$ in $E_{n}$ for all $\lambda \in(0,1]$ and $y \in \partial U_{n}$.

Then $F$ has a fixed point in $E$.

PROOF. Now (2.18) is immediate if we take for each $n \in N, H_{n}(x, \lambda)=\lambda F(x)$ for $(x, \lambda) \in \bar{U}_{n} \times[0,1]$. Our result follows from Theorem 2.15 .

Next we present a Krasnoselskii type result for weakly inward maps in the Fréchet space setting.

THEOREM 2.21. Let $E$ and $E_{n}$ be as described at the beginning of Section 2, $C$ a convex subset in $E$, and suppose that $U$ and $V$ are bounded pseudo-open subsets of $E$ with $0 \in U \subseteq \bar{U} \subseteq V$ and $F: Y \rightarrow 2^{E}$ with $Y \subseteq E$. Also assume that either $\bar{W}_{n}=\bar{V}_{n} \cap \bar{C}_{n} \subseteq Y_{n}$ for each $n \in N$ (here $W_{n}=V_{n} \cap \bar{C}_{n}$ ) or $\bar{W}_{n}$ is a subset of the closure of $Y_{n}$ in $E_{n}$ for each $n \in N$ (with $Y$ a closed subset of $E$ ). Also for each $n \in N$ assume that $F_{n}: \bar{W}_{n} \rightarrow 2^{E_{n}}$ and suppose that the following conditions are satisfied:

For each $n \in N$ the map $F_{n}: \bar{W}_{n} \rightarrow C K\left(E_{n}\right)$ is upper semicontinuous

condensing with $F_{n}(x) \cap \bar{I}_{\bar{C}_{n}}(x) \neq \emptyset$ for all $x \in \bar{W}_{n}$, where $\bar{W}_{n}$

denotes the closure of $W_{n}$ in $\bar{C}_{n}$.

For each $n \in N, y \notin \lambda F_{n} y$ in $E_{n}$ for all $\lambda \in[0,1]$ and $y \in \partial W_{n}$.

$\left\{\right.$ For each $n \in N$, there exists $v_{n} \in \bar{C}_{n} \backslash\{0\}$ with $x \notin F_{n} x+\delta v_{n}$ for all

$\left\{\begin{array}{l}\text { For each } n \in N \text {, the map } F_{n}(\cdot)+\mu v_{n}: \bar{\Omega}_{n} \rightarrow C K\left(E_{n}\right) \text { is weakly } \\ \text { inward with respect to } \bar{C}_{n} \text { for all } \mu \geq 0(\text { that is, } \\ \left.\left[F_{n}(x)+\mu v_{n}\right] \cap \bar{I}_{\bar{C}_{n}}(x) \neq \emptyset \text { for all } x \in \bar{\Omega}_{n}\right) .\end{array}\right.$

$\left\{\begin{array}{l}\text { For each } n \in\{2,3, \ldots\}, \text { if } y \in W_{n} \backslash \Omega_{n} \text { solves } y \in F_{n} y \text { in } E_{n} \text {, then } \\ j_{k} \mu_{k, n} j_{n}^{-1}(y) \in W_{k} \text { for } k \in\{1, \ldots, n-1\} .\end{array}\right.$ 
For any sequence $\left\{y_{n}\right\}_{n \in N}$ with $y_{n} \in W_{n} \backslash \Omega_{n}$ and $y_{n} \in F_{n} y_{n}$ in $E_{n}$ for $n \in N$ and for every $k \in N$ there exist a subsequence

$N_{k} \subseteq\{k+1, k+2, \ldots\}$, with $N_{k} \subseteq N_{k-1}$ for $k \in\{1,2, \ldots\}, N_{0}=N$, and $z_{k} \in \bar{W}_{k}$ with $j_{k} \mu_{k, n} j_{n}^{-1}\left(y_{n}\right) \rightarrow z_{k}$ in $E_{k}$ as $n \rightarrow \infty$ in $N_{k}$.

For every $k \in N$ and any subsequence $A \subseteq\{k, k+1, \ldots\}$, if $x \in \bar{C}_{n}$ is such that $x \in W_{n} \backslash \Omega_{n}$ for some $n \in A$ then there exists $\gamma>0$

(independent of $k$ and $n$ ) with $\left|j_{k} \mu_{k, n} j_{n}^{-1} x\right|_{k} \geq \gamma$.

$$
\left\{\begin{array}{l}
\text { If there exist } w \in Y \text { and a sequence }\left\{y_{n}\right\}_{n \in N} \text { with } y_{n} \in W_{n} \backslash \Omega_{n} \text { and } \\
y_{n} \in F_{n} y_{n} \text { in } E_{n} \text { such that for every } k \in N \text { there exists a subsequence } \\
S \subseteq\{k+1, k+2, \ldots\} \text { of } N \text { with } j_{k} \mu_{k, n} j_{n}^{-1}\left(y_{n}\right) \rightarrow j_{k} \mu_{k}(w) \text { in } E_{k} \\
\text { as } n \rightarrow \infty \text { in } S, \text { then } w \in F w \text { in } E .
\end{array}\right.
$$

Then $F$ has a fixed point in $E$.

ProOF. Fix $n \in N$. Now $\bar{C}_{n}$ is convex and $U_{n}, V_{n}$ are open bounded subsets of $E_{n}$ with $j_{n} \mu_{n}(0) \in U_{n} \subseteq V_{n}$. It just remains to show that $U_{n} \subseteq \bar{U}_{n} \subseteq V_{n}$. Of course since $U \subseteq \bar{U} \subseteq V$,

$$
U_{n}=j_{n} \mu_{n}(U) \subseteq j_{n} \mu_{n}(\bar{U}) \subseteq j_{n} \mu_{n}(V)=V_{n}
$$

and since $j_{n} \mu_{n}$ is continuous $U_{n} \subseteq j_{n} \mu_{n}(\bar{U}) \subseteq \overline{j_{n} \mu_{n}(U)}=\bar{U}_{n}$. Also we see that $\overline{\mu_{n}(U)} \subseteq \mu_{n}(V)$ (note that $\bar{U} \subseteq V$ ) so since $j_{n}$ is an isometry,

$$
\bar{U}_{n}=\overline{j_{n} \mu_{n}(U)}=j_{n} \overline{\mu_{n}(U)} \subseteq j_{n} \mu_{n}(V)=V_{n} .
$$

Theorem 1.14 guarantees that there exists $y_{n} \in W_{n} \backslash \Omega_{n}$ with $y_{n} \in F_{n} y_{n}$ in $E_{n}$. As in Theorem 2.2 there exist a subsequence $N_{1} \subseteq\{2,3, \ldots\}$ and $z_{1} \in \bar{W}_{1}$ with $j_{1} \mu_{1, n} j_{n}^{-1}\left(y_{n}\right) \rightarrow z_{1}$ in $E_{1}$ as $n \rightarrow \infty$ in $N_{1}$. Also $y_{n} \in W_{n} \backslash \Omega_{n}$ together with (2.26) yields $\left|j_{1} \mu_{1, n} j_{n}^{-1}\left(y_{n}\right)\right|_{1} \geq \gamma$ for $n \in N$ and so $\left|z_{1}\right|_{1} \geq \gamma$. Proceed inductively to obtain subsequences of integers

$$
N_{1} \supseteq N_{2} \supseteq \cdots \quad \text { where } N_{k} \subseteq\{k+1, k+2, \ldots\}
$$

and $z_{k} \in \bar{W}_{k}$ with $j_{k} \mu_{k, n} j_{n}^{-1}\left(y_{n}\right) \rightarrow z_{k}$ in $E_{k}$ as $n \rightarrow \infty$ in $N_{k}$. Note that $j_{k} \mu_{k, k+1} j_{k+1}^{-1} z_{k+1}=z_{k}$ in $E_{k}$ for $k \in\{1,2, \ldots\}$ and $\left|z_{k}\right|_{k} \geq \gamma$. Now essentially the same reasoning as in Theorem 2.2 (with Remark 2.5) guarantees the result.

REMARK 2.22. Condition (2.24) can be removed from the statement of Theorem 2.21.

REMARK 2.23. Condition (2.26) is only needed to guarantee that the fixed point $y$ satisfies $\left|j_{k} \mu_{k}(y)\right|_{k} \geq \gamma$ for $k \in N$. If we assume all the conditions in Theorem 2.21 except (2.26) then again $F$ has a fixed point in $E$ but the above property is not guaranteed.

We next present a Mönch type result using Theorem 1.15.

THEOREM 2.24. Let $E$ and $E_{n}$ be as described at the beginning of Section 2, $X \subseteq E$ and $F: Y \rightarrow 2^{E}$ with $\overline{\text { int } X_{n}} \subseteq Y_{n}$ for each $n \in N$ or $\overline{\text { int } X_{n}}$ is a subset of the closure 
of $Y_{n}$ in $E_{n}$ for each $n \in N$ (with $Y$ a closed subset of $E$ ). Also for each $n \in N$ assume that $F_{n}: \overline{\text { int } X_{n}} \rightarrow 2^{E_{n}}$ and suppose that the following conditions are satisfied.

$\left\{\begin{array}{l}\text { For each } n \in\{2,3, \ldots\} \text { if } y \in \text { int } X_{n} \text { solves } y \in F_{n} y \text { in } E_{n} \text { then } \\ j_{k} \mu_{k, n} j_{n}^{-1}(y) \in \text { int } X_{k} \text { for } k \in\{1, \ldots, n-1\} .\end{array}\right.$

$x_{0} \in \operatorname{pseudo-int}(X)$.

$\left\{\begin{array}{l}\text { For each } n \in N \text {, the map } F_{n}: \overline{\text { int } X_{n}} \rightarrow C K\left(E_{n}\right) \text { is upper } \\ \text { semicontinuous. }\end{array}\right.$

$\left\{\begin{array}{l}\text { For each } n \in N, M \subseteq \overline{\text { int } X_{n}} \text { with } M \subseteq \operatorname{co}\left(\left\{j_{n} \mu_{n}\left(x_{0}\right)\right\} \cup F_{n}(M)\right) \text { with } \\ \bar{M}=\bar{C} \text { and }\end{array}\right.$

$\left\{\begin{array}{l}\text { For each } n \in N, y \notin(1-\lambda) j_{n} \mu_{n}\left(x_{0}\right)+\lambda F_{n} y \text { in } E_{n} \text { for all } \lambda \in(0,1] \\ \text { and } y \in \partial \text { int } X_{n} .\end{array}\right.$

$\left\{\begin{array}{l}\text { For any sequence }\left\{y_{n}\right\}_{n \in N} \text { with } y_{n} \in \text { int } X_{n} \text { and } y_{n} \in F_{n} y_{n} \text { in } E_{n} \text { for } \\ n \in N \text { and for every } k \in N \text { there exist a subsequence } \\ N_{k} \subseteq\{k+1, k+2, \ldots\}, \text { with } N_{k} \subseteq N_{k-1} \text { for } k \in\{1,2, \ldots\}, N_{0}=N, \\ \text { and } z_{k} \in \overline{\text { int } X_{k}} \text { with } j_{k} \mu_{k, n} j_{n}^{-1}\left(y_{n}\right) \rightarrow z_{k} \text { in } E_{k} \text { as } n \rightarrow \infty \text { in } N_{k} .\end{array}\right.$

$\left\{\begin{array}{l}\text { If there exist } w \in Y \text { and a sequence }\left\{y_{n}\right\}_{n \in N} \text { with } y_{n} \in \text { int } X_{n} \text { and } \\ y_{n} \in F_{n} y_{n} \text { in } E_{n} \text { such that for every } k \in N \text { there exists a subsequence } \\ S \subseteq\{k+1, k+2, \ldots\} \text { of } N \text { with } j_{k} \mu_{k, n} j_{n}^{-1}\left(y_{n}\right) \rightarrow j_{k} \mu_{k}(w) \text { in } E_{k} \\ \text { as } n \rightarrow \infty \text { in } S, \text { then } w \in F w \text { in } E .\end{array}\right.$

Then $F$ has a fixed point in $E$.

REMARK 2.25. Condition (2.28) can be removed from the statement of Theorem 2.24 .

REMARK 2.26. Suppose that in Theorem 2.24 we replace (2.33) with the following condition.

$\left\{\begin{array}{l}\text { For any sequence }\left\{y_{n}\right\}_{n \in N} \text { with } y_{n} \in \operatorname{int} X_{n} \text { and } y_{n} \in F_{n} y_{n} \text { in } E_{n} \text { for } \\ n \in N \text { and for every } k \in N \text { there exist a subsequence } \\ N_{k} \subseteq\{k+1, k+2, \ldots\}, \text { with } N_{k} \subseteq N_{k-1} \text { for } k \in\{1,2, \ldots\}, N_{0}=N \\ \text { and } z_{k} \in \text { int } X_{k} \text { with } j_{k} \mu_{k, n} j_{n}^{-1}\left(y_{n}\right) \rightarrow z_{k} \text { in } E_{k} \text { as } n \rightarrow \infty \text { in } N_{k} .\end{array}\right.$

In addition, we assume that $F: Y \rightarrow 2^{E}$ with $\overline{\operatorname{int} X_{n}} \subseteq Y_{n}$ (or $\overline{\operatorname{int} X_{n}}$ is a subset of the closure of $Y_{n}$ in $E_{n}$ if $Y$ is a closed subset of $E$ ) for each $n \in N$ is replaced by $F: X \rightarrow 2^{E}$, and suppose that (2.34) is true with $w \in Y$ replaced by $w \in X$. Then the result in Theorem 2.24 is again true.

Also we have the following result for Mönch inward type maps (just apply Theorem 1.17 in this case).

THEOREM 2.27. Let $E$ and $E_{n}$ be as described at the beginning of Section 2, $C$ a convex subset in $E, V$ a pseudo-open bounded subset of $E, 0 \in V \cap C, F: Y \rightarrow 2^{E}$

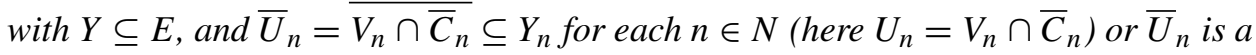


subset of the closure of $Y_{n}$ in $E_{n}$ (with $Y$ a closed subset of $E$ ). Also for each $n \in N$ assume that $F_{n}: \bar{U}_{n} \rightarrow 2^{E_{n}}$ and suppose that (2.5), (2.7), (2.8) and the following conditions hold.

$\left\{\begin{array}{l}\text { For each } n \in N, \text { the map } F_{n}: \bar{U}_{n} \rightarrow C K\left(E_{n}\right) \text { is upper semicontinuous } \\ \text { and } F_{n}\left(\bar{U}_{n}\right) \text { is bounded, where } \bar{U}_{n} \text { denotes the closure of } U_{n} \text { in } \bar{C}_{n} .\end{array}\right.$
$\left\{\begin{array}{l}\text { For each } n \in N, D \subseteq E_{n} \text { with } D \subseteq \operatorname{co}\left(\left\{j_{n} \mu_{n}(0)\right\} \cup F_{n}\left(D \cap U_{n}\right)\right) \text { and } \\ \bar{D}=\bar{B} \text { with } B \subseteq D \text { countable implies that } \bar{D} \cap U_{n} \text { is compact. }\end{array}\right.$

In addition, assume that (2.9) and (2.10) hold. Then F has a fixed point in E.

REMARK 2.28. Remarks 2.4 and 2.6 also hold in this situation.

Finally, we consider contractive type maps. First we consider single-valued maps (just apply Theorem 1.18).

THEOREM 2.29. Let $E$ and $E_{n}$ be as described at the beginning of Section 2, $X \subseteq E$ and $F: Y \rightarrow E$ with $\overline{\text { int } X_{n}} \subseteq Y_{n}$ for each $n \in N$ or int $X_{n}$ is a subset of the closure of $Y_{n}$ in $E_{n}$ for each $n \in N$ (with $Y$ a closed subset of $E$ ). Also for each $n \in N$ assume that $F_{n}: \overline{\operatorname{int} X_{n}} \rightarrow E_{n}$ and suppose that (2.28) and the following conditions are satisfied.

$0 \in \operatorname{pseudo-int}(X)$.

For each $n \in N, F_{n}\left(\overline{\operatorname{int} X_{n}}\right)$ is bounded.

For each $n \in N$, there exists a continuous nondecreasing function

$\left\{\phi_{n}:[0, \infty) \rightarrow[0, \infty)\right.$ satisfying $\phi_{n}(z)<z$ for $z>0$ such that

$\left|F_{n} x-F_{n} y\right|_{n} \leq \phi_{n}\left(|x-y|_{n}\right)$ for all $x, y \in \overline{\operatorname{int} X_{n}}$.

For each $n \in N, y \neq \lambda F_{n} y$ in $E_{n}$ for all $\lambda \in(0,1]$ and $y \in \partial$ int $X_{n}$.

Also assume that (2.33) (with $y_{n} \in F_{n} y_{n}$ replaced by $y_{n}=F_{n} y_{n}$ ) and (2.34) (with $y_{n} \in F_{n} y_{n}$ and $w \in F w$ replaced by $y_{n}=F_{n} y_{n}$ and $\left.w=F w\right)$ hold. Then $F$ has $a$ fixed point in $E$.

REMARK 2.30. Note that there is an analogue of Remarks 2.25 and 2.26 in this situation and also in the next.

THEOREM 2.31. Let $E$ and $E_{n}$ be as described at the beginning of Section 2, $X \subseteq E$ and $F: Y \rightarrow 2^{E}$ with $\overline{\text { int } X_{n}} \subseteq Y_{n}$ for each $n \in N$ or int $X_{n}$ is a subset of the closure of $Y_{n}$ in $E_{n}$ for each $n \in N$ (with $Y$ a closed subset of $E$ ). Also for each $n \in N$ assume that $F_{n}: \overline{\operatorname{int} X_{n}} \rightarrow 2^{E_{n}}$ and suppose that (2.28), (2.37) and the following conditions are satisfied.

For each $n \in N, F_{n}\left(\overline{\operatorname{int} X_{n}}\right)$ is bounded.

For each $n \in N, F_{n}: \overline{\text { int } X_{n}} \rightarrow C\left(E_{n}\right)$ and there exists a continuous

strictly increasing function $\phi_{n}:[0, \infty) \rightarrow[0, \infty)$ satisfying $\phi_{n}(z)<z$

for $z>0$ such that $H_{n}\left(F_{n} x, F_{n} y\right) \leq \phi_{n}\left(|x-y|_{n}\right)$ for all
$x, y \in \overline{\operatorname{int} X_{n}}$. 


$$
\left\{\begin{array}{l}
\text { For each } n \in N, \text { the map } \Phi_{n}:[0, \infty) \rightarrow[0, \infty) \text { given by } \\
\Phi_{n}(x)=x-\phi_{n}(x) \text { is strictly increasing, } \\
\Phi_{n}^{-1}(a)+\Phi_{n}^{-1}(b) \leq \Phi_{n}^{-1}(a+b) \text { for } a, b \geq 0, \text { with } \sum_{i=0}^{\infty} \phi_{n}^{i}(t)<\infty \text { for } \\
t>0 \text { and } \sum_{i=1}^{\infty} \phi_{n}^{i}(x-\phi(x)) \leq \phi_{n}(x) \text { for } x>0 .
\end{array}\right.
$$

For each $n \in N, y \notin \lambda F_{n} y$ in $E_{n}$ for all $\lambda \in(0,1]$ and $y \in \partial$ int $X_{n}$.

Also assume that (2.33) and (2.34) hold. Then F has a fixed point.

\section{References}

[1] R. P. Agarwal, J. Dshalalow and D. O'Regan, 'Fixed point and homotopy results for generalized contractive maps of Reich type', Appl. Anal. 82 (2003), 329-350.

[2] R. P. Agarwal, J. H. Dshalalow and D. O'Regan, 'Fixed point theory for Mönch type maps defined on closed subsets of Fréchet spaces: the projective limit approach', Int. J. Math. Math. Sci. 17 (2005), 2775-2782.

[3] R. P. Agarwal, J. H. Dshalalow and D. O'Regan, 'Leray-Schauder principles for inward Kakutani Mönch type maps', Nonlinear Funct. Anal. Appl. 10 (2005), 325-330.

[4] R. P. Agarwal, M. Frigon and D. O'Regan, 'A survey of recent fixed point theory in Fréchet spaces', in: Nonlinear Analysis and Applications: to V. Lakshmikantham on his 80th Birthday, Vol. 1 (Kluwer Academic Publishers, Dordrecht, 2003), pp. 75-88.

[5] R. P. Agarwal and D. O'Regan, 'Fixed point theory for weakly inward Kakutani maps: the projective limit approach', Proc. Amer. Math. Soc. 135 (2007), 417-426.

[6] K. Deimling, Multivalued Differential Equations (Walter de Gruyter, Berlin, 1992).

[7] L. V. Kantorovich and G. P. Akilov, Functional Analysis in Normed Spaces (Pergamon Press, Oxford, 1964).

[8] D. O'Regan, 'Fixed point theorems for nonlinear operators', J. Math. Anal. Appl. 202 (1996), 413-432.

[9] D. O'Regan, 'A continuation theory for weakly inward maps', Glasg. Math. J. 40 (1998), 311-321.

[10] D. O'Regan, 'Homotopy and Leray-Schauder type results for admissible inward multimaps', J. Concr. Appl. Math. 2 (2004), 67-76.

[11] D. O'Regan, 'Leray-Schauder results for inward acyclic and approximable maps defined on Fréchet space', Appl. Math. Lett. 19 (2006), 976-982.

[12] D. O'Regan, 'Fixed point theory in Fréchet spaces for Mönch inward and contractive Urysohn type operators', East Asian Math. J. 24 (2008), 233-249.

[13] D. O'Regan and R. Precup, 'Fixed point theory for set valued maps and existence principles for integral inclusions', J. Math. Anal. Appl. 245 (2000), 594-612.

RAVI P. AGARWAL, Department of Mathematical Science, Florida Institute of Technology, Melbourne, Florida 32901, USA e-mail: agarwal@fit.edu

DONAL O'REGAN, Department of Mathematics, National University of Ireland, Galway, Ireland e-mail: donal.oregan@nuigalway.ie 